\title{
On the discrete eigenvalues of the many-particle system
}

\author{
By
}

Jun UCHIYAMA*

\section{$\$ 1$. Introduction}

Let us consider a system in a static magnetic field which consists of $N$ electrons and $M$ infinitely heavy nuclei. Then the Schrc̈dinger operator in the nonrelativistic quantum mechanics becomes,

$$
\begin{aligned}
H=\sum_{k=1}^{N}\left\{\sum _ { \nu = 0 } ^ { 2 } \frac { \hbar ^ { 2 } } { 2 \mu _ { k } } \left(i \frac{\partial}{\partial x_{3 k-\nu}}\right.\right. & \left.\left.+\frac{e_{k}}{c} A\left(\boldsymbol{r}_{k}\right)\right)^{2}-\sum_{j=1}^{M} \frac{z_{j} e_{k}}{\left|\boldsymbol{r}_{k}-\boldsymbol{a}_{j}\right|}\right\} \\
& +\sum_{\substack{k, h=1 \\
k<h}}^{N} \frac{\boldsymbol{e}_{k} e_{h}}{\left|\boldsymbol{r}_{k}-\boldsymbol{r}_{h}\right|} .
\end{aligned}
$$

Recently J̈̈rgens [1] has shown that the essential spectrum of $H$ is $[\mu, \infty)$, where $\mu \leq 0$, and Žislin [2] has shown that the operator of the form

$$
-\sum_{k, h=1}^{N} \sum_{\nu=0}^{2} a_{k h} \frac{\partial^{2}}{\partial x_{3 k-\nu} \partial x_{3 k-\nu}}-\sum_{k=1}^{N} \sum_{j=1}^{M} \frac{b_{k j}}{\left|\boldsymbol{r}_{k}-\boldsymbol{a}_{j}\right|}+\sum_{\substack{k, n=1 \\ h<h}}^{N} \frac{c_{k h}}{\left|\boldsymbol{r}_{k}-\boldsymbol{r}_{h}\right|},
$$

where $\left(a_{k h}\right)$ is a constant positive matrix and $\sum_{j=1}^{M} b_{k j}>\sum_{\substack{h=1 \\ h \neq k}}^{N} c_{k h}$ for each $k$, has a countably infinite number of discrete eigenvalues.

Making use of Žislin's method, we get the same result for a many-particle system which composes a positive ion, a neutral atom or a neutral molecule in a static magnetic field. At the same time, it will be seen how the decreasing orders at infinity

Received May 5, 1966.

Communicated by S. Mizohata.

* Department of Mathematics, Kyoto University 
of the attractive potentials and the vector potentials influence the number of the discrete eigenvalues. Our result is a kind of extension of the well-known fact that the operator in $L^{2}\left(R^{3}\right)$

$$
-\Delta+c(x)
$$

where $c(x) \leqq-\frac{1}{4-\varepsilon} \cdot \frac{1}{|\boldsymbol{r}|^{2}}$ for $|\boldsymbol{r}| \geqq R_{0}$ and it converges uniformly to zero when $|\boldsymbol{r}|$ tends to infinity, has countably infinite discrete eigenvalues.

\section{\$2. Statement of the theorem}

We denote by $R^{m}$ the $m$-dimensional Euclidean space, by $\mathbf{R}_{k}^{3 N}$ the 3-dimensional Euclidean space with variables $\boldsymbol{r}_{k}=\left(x_{3 k-2}, x_{3 k-1}\right.$, $\left.x_{3 k}\right)$ and by $R_{\left(k^{\prime}\right)}^{3 N}$ the $(3 N-3)$-dimensional Euclidean space with variables $r_{1}, \cdots, r_{k-1}, r_{k+1}, \cdots, r_{N}$.

Let us consider the Schrc̈dinger operator of the form

$$
H=\sum_{k=1}^{N}\left\{\sum_{\nu=0}^{2}\left(i \frac{\partial}{\partial x_{3 k-\nu}}+b_{3 k-\nu}\left(\boldsymbol{r}_{k}\right)\right)^{2}+q_{k}\left(\boldsymbol{r}_{k}\right)\right\}+\sum_{\substack{k, h=1 \\ k, h}}^{N} P_{k h}\left(\boldsymbol{r}_{k}, \boldsymbol{r}_{h}\right) .
$$

For each term of this operator, we assume that

(c-1) $b_{3 k-\nu}, q_{k}$ and $P_{k h}$ are real-valued functions,

(c-2) $b_{3 k-\nu}^{2}\left(\boldsymbol{r}_{k}\right), q_{k}\left(\boldsymbol{r}_{k}\right)$ and $\frac{\partial b_{3 k-\nu}}{\partial x_{3 k-\nu}}\left(\boldsymbol{r}_{k}\right)$ belong to $\mathrm{L}_{\mathrm{kc}}^{2}\left(\mathbf{R}_{k}^{3 N}\right)$,

(c-3) there exist some $\beta(0<\beta<2), \gamma\left(0<\gamma<\frac{3}{2}\right), \quad \beta^{\prime}$ (max $\left.(\beta, \gamma)<\beta^{\prime}<3\right), c_{k}>0, d_{k h}>0, \varepsilon>0, R_{2}\left(0<R_{2}<1\right)$ and sufficiently large $R_{0}>0, R_{1}>0$ such that

$$
\begin{aligned}
& q_{k}\left(\boldsymbol{r}_{k}\right) \leqq-\frac{c_{k}}{\left|\boldsymbol{r}_{k}\right|^{\beta}} \\
& \text { for }\left|r_{k}\right| \geqq R_{0},{ }^{1)} \\
& \left|b_{3 k-\nu}\left(\boldsymbol{r}_{k}\right)\right| \leqq \frac{\text { const }}{\left|\boldsymbol{r}_{k}\right|^{\frac{\beta}{2}+\varepsilon}} \quad \text { for } \quad\left|\boldsymbol{r}_{k}\right| \geqq R_{0} \text {, } \\
& 0 \leqq P_{k h}\left(\boldsymbol{r}_{k}, \boldsymbol{r}_{h}\right) \begin{cases}\leqq \frac{d_{k h} R_{1}^{\beta^{\prime}-\beta} R_{2}^{2-\beta^{\prime}}}{\left|\boldsymbol{r}_{k}-\boldsymbol{r}_{h}\right|^{r}} & \text { for }\left|\boldsymbol{r}_{k}-\boldsymbol{r}_{k}\right| \leqq R_{2}, \\
\leqq \frac{d_{k h} R_{1}^{\beta^{\prime}-\beta}}{\left|\boldsymbol{r}_{k}-\boldsymbol{r}_{h}\right|^{\beta^{\prime}}} & \text { for } R_{2} \leqq\left|\boldsymbol{r}_{k}-\boldsymbol{r}_{h}\right| \leqq R_{1},\end{cases}
\end{aligned}
$$


On the discrete eigenvalues of the many-particle system 119

$$
\left\lfloor\leqq \frac{d_{k h}}{\left|r_{k}-r_{h}\right|^{\beta}} \quad \text { for }\left|r_{k}-r_{h}\right| \geqq R_{1} \cdot{ }^{1)}\right.
$$

(2.5) $q_{k}\left(\boldsymbol{r}_{k}\right)$ and $\frac{\partial b_{3 k-\nu}}{\partial x_{3 k-\nu}}\left(\boldsymbol{r}_{k}\right)$ converge uniformly to zero, when $\left|\boldsymbol{r}_{k}\right|$ tends to infinity. And for convenience, put $P_{k h}=P_{h k}$ for $k>h$.

$$
\text { (c-4) } \quad c_{k}>\sum_{\substack{h=1 \\ k \neq h}}^{N} d_{k h} .
$$

Under these assumptions we can prove the following theorems.

Theorem 1. The Schrëdinger operator $H$ of the form (2.1) has the following properties;

i) the essential spectrum of $H$ is $[\mu, \infty)$, where $\mu=0$ for $N=1$ or $\mu<0$ for $N \geqq 2$.

ii) there exist a countably infinite number of discrete eigenvalues and they have the only limit point at $\mu$.

Theorem 2. When the conditions (2.2) and (2.3) are satisfied only in a cone $\mathrm{C}_{k}$ whose vertex is the origin of $\mathrm{R}_{\mathrm{r}}^{3 . N}$, then the statement of Theorem 1 is still true. In this case, however, we still assume that outside of the cone $b_{3 k-\nu}\left(r_{k}\right)$ converges uniformly to zero, when $\left|\boldsymbol{r}_{k}\right|$ tends to in finity. ${ }^{2)}$

Theorem 3. If the condition (c-3) is satisfied by $\beta=2$, we have the same assertion as that of Theorem 1 by replacing the condition (c-4) by the following one

$$
\left(\mathrm{c}-4^{\prime}\right) \quad c_{k}-\sum_{\substack{h=1 \\ h \neq h}}^{N} d_{k h}>\frac{1}{4} .
$$

Remark 1. The condition (2.4) is satisfied if one takes $P_{k h}$ to be the following:

$$
P_{k h h}\left(\boldsymbol{r}_{k}, \boldsymbol{r}_{h}\right)=f\left(\boldsymbol{r}_{k}-\boldsymbol{r}_{h}\right)
$$

where

i) $f(\boldsymbol{r}) \geqq 0 \quad\left(\boldsymbol{r} \in R^{3}\right)$

1) We write $\left|\boldsymbol{r}_{k}\right|=\left(\sum_{\nu=0}^{2} x_{3 k-\nu}^{2}\right)^{\frac{1}{2}}$ and $\left|\boldsymbol{r}_{k}-\boldsymbol{r}_{h}\right|=\left(\sum_{\nu=0}^{2}\left(x_{3 h-\nu}-x_{3 h-\nu}\right)^{2}\right)^{\frac{1}{2}}$.

2) This supplementary condition is imposed so that Lemma 2 may hold. 
ii) $f(\boldsymbol{r})=O\left(\frac{1}{|\boldsymbol{r}|^{1.5-\varepsilon}}\right)$ in the neighborhood of the origin,

iii) $f(\boldsymbol{r})$ is bounded except for the neighborhood of the origin of $R^{3}$,

iv) $f(\boldsymbol{r}) \leqq \frac{d_{k h}}{|\boldsymbol{r}|^{\beta}}$ for $|\boldsymbol{r}| \geqq R_{0}$.

\section{$\S 3$. Some lemmas}

In the first place let us introduce the spaces of functions, $\mathrm{C}_{0}^{r}\left(\mathrm{R}^{m}\right)$ and $\mathfrak{D}_{\mathrm{L}^{2}}^{2}\left(\mathbf{R}^{m}\right)$. The former is the space of all $\mathrm{C}^{\infty}$ functions with compact support, and the latter the completion of the space $\mathrm{C}_{0}^{\infty}\left(\mathrm{R}^{m}\right)$ with the norm

$$
\|f\|_{2, \mathrm{~L}^{2}\left(\mathrm{R}^{m}\right)}=\left(\sum_{i, j=1}^{m}\left\|\frac{\partial^{2} f}{\partial x_{i} \partial x_{j}}\right\|_{\mathrm{L}^{2}\left(\mathrm{R}^{m}\right)}^{2}+\sum_{i=1}^{m}\left\|\frac{\partial f}{\partial x_{i}}\right\|_{\mathrm{L}^{2}\left(\mathrm{R}^{m}\right)}^{2}+\|f\|_{\mathrm{L}^{2}\left(\mathrm{~K}^{m}\right)}^{2}\right)^{\frac{1}{2} \cdot{ }^{3)}}
$$

Under the conditions $(\mathrm{c}-1) \sim(\mathrm{c}-3)$ the following two lemmas hold. (see Jörgens [1]). ${ }^{4}$

Lemma 1. If the domain of $H$ is $\mathfrak{D}_{\mathrm{L}^{2}}^{2}\left(\mathbf{R}^{3 N}\right)$, then $H$ is a lowerbounded selfadjoint operator in $L^{2}\left(\mathrm{R}^{3 N}\right)$.

Lemma 2. The essential spectrum of $H$ is $[\mu, \infty]$, where

$$
\mu=\left\{\begin{array}{lr}
0, & \text { when } N=1, \\
\min _{1 \leq i \leq N} \inf \left\{\left(H_{(i)} \varphi, \varphi\right)_{L^{2}\left(\mathrm{R}_{(i)}^{3 V}\right)} ; \varphi \in \mathscr{D}_{\mathrm{L}^{2}}^{2}\left(\mathrm{R}_{(i)}^{3 N}\right),\right. & \left.\|\varphi\|_{\mathrm{L}^{2}\left(\mathrm{R}_{(i)}^{3 N}\right)}=1\right\}, \\
\text { when } N \geqq 2,
\end{array}\right.
$$

(3.2) $\quad H_{(\ell)}=\sum_{\substack{k=1 \\ k \neq i}}^{N}\left\{\sum_{\nu=0}^{2}\left(i \frac{\partial}{\partial x_{3 k-\nu}}+b_{3 k-\nu}\left(\boldsymbol{r}_{k}\right)\right)^{2}+q_{k}\left(\boldsymbol{r}_{\kappa}\right)\right\}+\sum_{\substack{k, h=1 \\ k<h, k, h \neq i}}^{N} P_{k \hbar}\left(\boldsymbol{r}_{k}, \boldsymbol{r}_{h}\right)$.

Let us prove the following lemma.

Lemma 3. Let $N \geqq 2$. If $\varphi \in \mathrm{L}^{2}\left(\mathbf{R}_{(i)}^{3 \downarrow}\right), g \in \mathrm{C}_{0}^{\infty}\left(\mathbf{R}_{\imath}^{3 N}\right)$ and $0<\gamma<3$ are given, so we have

3) We write $\int_{\mathrm{R}^{m}} f(x) \overline{g(x)} d x=(f, g) \mathrm{L}^{2}\left(\mathrm{R}^{m}\right)$ and $\|f\|_{\mathrm{L}^{2}\left(\mathrm{R}^{m}\right)}=(f, f)_{\mathrm{L}^{2}\left(\mathrm{R}^{m}\right)}^{\frac{1}{2}}$.

4) As for $\frac{\partial b_{3 r-\nu}}{\partial x_{3 i-\nu}}$ Jörgens has assumed that $\sum_{\nu=0}^{2} \frac{\partial b_{3 k-\nu}}{\partial x_{3 k-\nu}}=0$ in distribution sense in place of the conditions (C-2) and (2-5). But following his proof, we can easily get the same results as his under our conditions. 


$$
\int \frac{|\rho g|^{2}}{\left|l r_{j}-r_{i}\right|^{r}} d x \leqq \text { const. , } \quad(j \neq i) .
$$

where $l$ is a real parameter and const. is some constant independent of $l .{ }^{5}$ )

PROOF. Putting $d r_{i}=d x_{3 i-2} d x_{3 i-1} d x_{3 i}$ and

$$
d \widehat{\mathbf{r}}_{i}=d \boldsymbol{r}_{1} \cdots d \boldsymbol{r}_{i-1} d \boldsymbol{r}_{i+1} \cdots d \boldsymbol{r}_{N}
$$

we have

$$
\begin{aligned}
\int \frac{|\varphi g|^{2}}{\left|l \boldsymbol{r}_{j}-\boldsymbol{r}_{i}\right|^{\prime}} d x= & \int_{\mathrm{R}_{(i)}^{3 N}}|\varphi|^{2} d \widehat{\mathbf{r}}_{i} \int_{\left|l \boldsymbol{r}_{j}-\boldsymbol{r}_{i}\right| \leqq 1} \frac{|g|^{2}}{\left|l \boldsymbol{r}_{j}-\boldsymbol{r}_{i}\right|^{r}} d \boldsymbol{r}_{i} \\
& +\int_{\mathrm{R}_{(i)}^{3 N}}|\varphi|^{2} d \widehat{\mathbf{r}}_{i} \int_{\left|l \boldsymbol{r}_{j}-\boldsymbol{r}_{i}\right| \geqq 1} \frac{|g|^{2}}{\left|l \boldsymbol{r}_{j}-\boldsymbol{r}_{i}\right|^{\gamma}} d \boldsymbol{r}_{i} \\
\leqq & \frac{4 \pi}{3-\gamma} \cdot \max _{\boldsymbol{r}_{i} \in \mathrm{R}_{i}^{3 N}}|g|^{2} \cdot\|\varphi\|_{\mathrm{L}^{2}\left(\mathrm{R}_{(2)}^{3 N}\right)}^{2 N}+\|g \varphi\|^{2} .
\end{aligned}
$$

Now we shall prove a lemma which is an extension of Lemma 7.1 of Žislin [2]. It plays an important role in showing the existence of the discrete eigenvalues of $H$.

Lemma 4. Let $N \geqq 2$. For any functions $\varphi \equiv \mathrm{L}^{2}\left(\mathrm{R}_{(i)}^{3 \vee}\right), g \in$ $\mathrm{C}_{0}^{\infty}\left(\mathrm{R}_{i}^{3 N}\right)$ and for $0<\gamma<3$, we have

$$
\lim _{l \rightarrow 0} \int \frac{|\varphi g|^{2}}{\left|l \boldsymbol{r}_{j}-\boldsymbol{r}_{i}\right|^{2}} d x=\int \frac{|\varphi g|^{2}}{\left|\boldsymbol{r}_{i}\right|^{r}} d x \quad(i, j=1, \cdots, N ; i \neq j) .{ }^{6)}
$$

PROOF. We put $\gamma=3-3 \varepsilon(0<\varepsilon<1)$, choose $M$ lagre enough to satisfy $\varepsilon M^{-1}<1-\varepsilon$ and $M \geqq \frac{1}{2}$, and then put $\theta=\varepsilon M^{-1}$.

Let $p^{\prime}=(3-\varepsilon) \cdot(3-3 \varepsilon-\theta)^{-1}$ and $p=(3-\varepsilon) \cdot(2 \varepsilon+\theta)^{-1}$. Then $p$ and $p^{\prime}$ satisfy the equality $\frac{1}{p}+\frac{1}{p^{\prime}}=1$ and the inequalities, $p^{\prime}(3-3 \varepsilon-\theta)<3,2 p \theta<3$.

Thus by virtue of Lemma 3 and the inequalities $a^{\alpha}+b^{\alpha}$ $\leqq$ const $(a+b)^{\alpha} \leqq$ const $\left(a^{\alpha}+b^{\alpha}\right)(\alpha>0) ; \quad\left|a^{\alpha}-b^{\alpha}\right| \leqq|a-b|^{\alpha}(0 \leqq \alpha \leqq 1)$.

5) Hereafter we write simply $\int f(x) d x$ in place of $\int_{R^{3 . v}} f(x) d x$, and denote by $(f, g)$ the integral $\int_{\mathrm{R}^{3 N}} f(x) \overline{\varepsilon(x)} d x$, finally $\|f\|=(f, f)^{\frac{1}{3}}$.

6) In the case $\gamma=1, Z$ islin [2] has shown this result. 
we have

$$
\begin{aligned}
& \left.\left|\int\left\{\frac{1}{\left|l r_{j}-r_{i}\right|^{3-3 \varepsilon}}-\frac{1}{\left|r_{i}\right|^{3-3 \varepsilon}}\right\}\right| \rho g\right|^{2} d x \mid \\
& \leqq \int \frac{1}{\left|l \boldsymbol{r}_{j}-\boldsymbol{r}_{i}\right|^{1-\varepsilon}}-\frac{1}{\left|\boldsymbol{r}_{i}\right|^{1-\varepsilon}} \mid\left\{\frac{1}{\left|l \boldsymbol{r}_{j}-\boldsymbol{r}_{i}\right|^{2-2 \varepsilon}}\right. \\
& \left.+\frac{1}{\left|l r_{j}-r_{i}\right|^{1-\varepsilon}} \cdot \frac{1}{\left|r_{i}\right|^{1-s}}+\frac{1}{\left|r_{i}\right|^{2-2 s}}\right\}|\varphi g|^{2} d x \\
& \leqq \text { const } \int\left|\frac{1}{\left|l r_{j}-r_{i}\right|}-\frac{1}{\left|\boldsymbol{r}_{i}\right|}\right|^{1-\varepsilon}\left\{\frac{1}{\left|l \boldsymbol{r}_{j}-\boldsymbol{r}_{i}\right|}\right. \\
& \left.+\frac{1}{\left|r_{i}\right|}\right\}^{2-2 s}|\rho g|^{2} d x \\
& \leqq \text { const } \int\left|\frac{1}{\left|l r_{j}-r_{i}\right|}-\frac{1}{\left|r_{i}\right|}\right|^{\theta}\left\{\frac{1}{\left|l r_{j}-r_{i}\right|}\right. \\
& \left.+\frac{1}{\left|\boldsymbol{r}_{i}\right|}\right\}^{1-s-\theta}\left\{\frac{1}{\left|l \boldsymbol{r}_{j}-\boldsymbol{r}_{i}\right|}+\frac{1}{\left|\boldsymbol{r}_{i}\right|}\right\}^{2-2 s}|\varphi g|^{2} d x \\
& \leqq \operatorname{const}\left(\int\left|\frac{1}{\left|l \boldsymbol{r}_{j}-\boldsymbol{r}_{\boldsymbol{i}}\right|}-\frac{1}{\left|\boldsymbol{r}_{i}\right|}\right|^{p \theta}|\rho g|^{2} d x\right)^{p} \text {. } \\
& \left(\int\left(\frac{1}{\left|l \boldsymbol{r}_{j}-\boldsymbol{r}_{i}\right|}+\frac{1}{\left|\boldsymbol{r}_{i}\right|}\right)^{\boldsymbol{p}(3-3 \varepsilon-\theta)}|\varphi g|^{2} d x\right)^{\frac{1}{p^{\prime}}} \\
& \leqq \operatorname{const}\left(\int\left|\frac{1}{\left|l \boldsymbol{r}_{j}-\boldsymbol{r}_{i}\right|}-\frac{1}{\left|\boldsymbol{r}_{i}\right|}\right|^{\boldsymbol{p} \vartheta}|\varphi g|^{2} d x\right)^{\frac{1}{p}} \text {. } \\
& \left(\int \frac{|\varphi g|^{2}}{\left|l r_{j}-r_{i}\right|^{p^{\prime}(3-3 \varepsilon-\theta)}} d x+\int \frac{|\varphi g|^{2}}{\left|r_{i}\right|^{p^{\prime}(3-3 \varepsilon-\theta)}} d x\right)^{\frac{1}{p^{\prime}}} \\
& \leqq \text { const }\left(\int\left|\frac{1}{\left|l r_{j}-r_{i}\right|}-\frac{1}{\left|r_{i}\right|}\right|^{p g}|\rho g|^{2} d x\right)^{\frac{1}{p}} \cdot{ }^{7)}
\end{aligned}
$$

Now for any $\eta>0$, there exist positive constants $N_{\eta}$ and $l_{\eta}$ such that

$$
\int_{r \geqq N}|\varphi g|^{2} d x<\eta^{2}, \quad \text { where } \quad r=\left(\sum_{k=1}^{N}\left|r_{k}\right|^{2}\right)^{\frac{1}{2}}
$$

and for all $r \leqq N_{\eta}$ and for any $l$, satisfing $|l|<l_{\eta}$,

$$
|| \boldsymbol{r}_{i}|-| l \boldsymbol{r}_{j}-\boldsymbol{r}_{i}||^{p_{\theta}} \leqq\left|l \boldsymbol{r}_{j}\right|^{p_{\theta}}<\eta
$$

hold. Then the right member of the inequality (3.3) is estimated as follows,

7) In the sequel, const. signifies some constant independent of a parameter $l$. 


$$
\begin{aligned}
& \text { (right member) })^{p} \leqq \text { const }\left\{\int_{r \leqq N_{\eta}} \frac{|| \boldsymbol{r}_{i}|-| l \boldsymbol{r}_{j}-\boldsymbol{r}_{i}||^{p \theta}}{\left|l \boldsymbol{r}_{j}-\boldsymbol{r}_{i}\right|^{p}\left|\boldsymbol{r}_{i}\right|^{p \theta}}|\rho g|^{2} d x\right. \\
& \left.+\int_{r \geqq N_{\eta}} \frac{|\varphi g|^{2}}{\left|l r_{j}-r_{i}\right|^{p \theta}} d x+\int_{r \geqq N_{\eta}} \frac{|\varphi g|^{2}}{\left|r_{i}\right|^{p \theta}} d x\right\} \\
& \leqq \text { const }\left\{\eta\left(\int_{r \leqq N_{\eta}} \frac{|\varphi g|^{2}}{\left|l r_{j}-r_{i}\right|^{2 p^{\theta}}} d x\right)^{\frac{1}{2}}\left(\int_{r \leqq N_{\eta}} \frac{|\varphi g|^{2}}{\left|r_{i}\right|^{2 p^{2}}} d x\right)^{\frac{1}{2}}\right. \\
& +\left(\int_{r \geqq N_{\eta}} \frac{|\varphi g|^{2}}{\left|l r_{j}-r_{i}\right|^{2 p \theta}} d x\right)^{\frac{1}{2}}\left(\int_{r \geqq N_{\eta}}|\varphi g|^{2} d x\right)^{\frac{1}{2}} \\
& \left.+\left(\int_{r \geqq N_{\eta}} \frac{|\varphi g|^{2}}{\left|\boldsymbol{r}_{i}\right|^{2 p_{\theta}}} d x\right)^{\frac{1}{2}}\left(\int_{r \geqq N_{\eta}}|\varphi g|^{2} d x\right)^{\frac{1}{2}}\right\} \\
& \leqq \text { const } \eta
\end{aligned}
$$

which proves the assertion.

\section{$\S 4$. Proof of the theorems}

At first we can assume $\gamma>\frac{\beta}{2}$ in the condition (2.4), for $\beta$ is smaller than 2 and $\gamma$ can be chosen as close to $\frac{3}{2}$ as we require. Now we divide the proof of Theorem 1. in several steps.

1-st step. We shall show that the lower limit of the spectrum is a discrete eigenvalue in the case $N=1$. For this purpose we have only to show $(H \varphi, \varphi)<0$ for some function $\phi \in \mathfrak{D}_{\mathrm{L}^{2}}^{2}\left(\mathbb{R}^{3}\right)$, since the essential spectrum of $H$ is $[0, \infty)$.

Now there exists some function with the properties;

$$
\begin{aligned}
& g_{1}\left(x_{1}, x_{2}, x_{3}\right) \in \mathrm{C}_{0}\left(\mathrm{R}^{3}\right), \quad\left\|g_{1}\right\|_{\mathrm{L}^{2} \mathrm{R} 3}=1,
\end{aligned}
$$

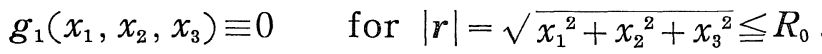

Let $g_{l}(x)=l^{3} g_{1}\left(l x_{1}, l x_{2}, l x_{3}\right)(1 \geqq l>0)$, then we have $\left\|g_{i}\right\|_{\mathrm{L} 2(\mathrm{R} 3)}=1$. Thus

$$
\begin{aligned}
\left(H g_{\imath}, g_{\imath}\right) \leqq & \left\|\left|\operatorname{grad} g_{\imath}\right|\right\|^{2}+2 \sum_{\nu=1}^{3} \int\left|b_{\nu} g_{\imath} \frac{\partial g_{l}}{\partial x_{\nu}}\right| d x \\
& +\sum_{\nu=1}^{3} \int b_{\nu}^{2}\left|g_{l}\right|^{2} d x+\left(q_{1} g_{l}, g_{l}\right) \\
\leqq & \operatorname{const}\left(l^{2}+l^{1+\frac{\beta}{2}+\varepsilon}+l^{\beta+2 \varepsilon}\right)-\operatorname{const} l^{\beta} .{ }^{8)}
\end{aligned}
$$

8) $\quad|\operatorname{grad} f|=\left(\sum_{\nu=1}^{3}\left|\frac{\partial f}{\partial x_{\nu}}\right|^{2}\right)^{\frac{1}{2}}$. 
Therefore there exists some $l_{0}\left(1 \geqq l_{0}>0\right)$ such that $\left(H g_{l_{0}}\right.$, $\left.g_{l_{0}}\right)<0$, which shows the assertion.

$2-n d$ step. Let us show that the assertion in the 1-st step is also true in the case $N \geqq 2$. First ws assume that there exists at least one discrete eigenvalue for the system composed of $N-1$ particles. Then by virtue of Lemma 2 there exists an operator $H_{\left(i_{0}\right)}$ in $\mathrm{L}^{2}\left(\mathrm{R}_{(i,)}^{3 N}\right)$ such that

$$
\mu=\inf \left\{\left(H_{\left(i_{\nu}\right)} \varphi, \varphi\right)_{L^{2}\left(R_{\left(i_{0}\right)}^{3 \mathrm{~V}}\right)} ; \varphi \in \mathfrak{D}_{\mathrm{L}^{2}}^{2}\left(\mathrm{R}_{\left(i_{0}\right)}^{3 N}\right),\|\varphi\|_{L^{2}\left(\mathrm{R}_{\left(i_{0}\right)}^{3 \mathrm{~V}}\right)}=1\right\} .
$$

By assumption, $\mu$ is a discrete eigenvalue of $H_{\left(i_{0}\right)}$. Thus there exists some function $\varphi_{0}$ such that

$$
\varphi_{0} \in \mathcal{D}_{L^{2}}^{2}\left(R_{\left.i_{0}\right)}^{3 \mathrm{v}}\right), \quad\left\|\varphi_{0}\right\|_{L^{2}\left(\mathrm{R}_{\left(i_{0}\right)}^{3 \mathrm{v}}\right)}=1 \text { and } H_{\left(i_{0}\right\rangle} \varphi_{0}=\mu \varphi_{0} .
$$

Putting $\psi_{l}=\varphi_{0}\left(\hat{\mathbf{r}}_{i_{0}}\right) g_{l}\left(r_{i_{0}}\right)$, we have $\psi_{l} \in \mathcal{D}_{\mathrm{L}^{2}}^{2}\left(\mathbf{R}^{3 N}\right),\left\|\psi_{\imath}\right\|=1$ and

$$
\begin{aligned}
& \left(H \psi_{l}, \psi_{l}\right)=\left(H_{\left(i_{\nu}\right)} \varphi_{0} g_{l}, \varphi_{0} g_{l}\right)+\sum_{\nu=0}^{2}\left(\left(i \frac{\partial}{\partial x_{3 i_{j-\nu}}}\right.\right. \\
& \left.\left.+b_{3 i_{\nu}-\nu}\left(r_{i_{0}}\right)\right)^{2} \varphi_{0} g_{l}, \varphi_{0} g_{l}\right)+\left(q_{i_{0}} \varphi_{0} g_{l}, \varphi_{0} g_{l}\right) \\
& +\sum_{\substack{k=1 \\
k \neq l_{0}}}^{N}\left(P_{i_{\iota} k} \varphi_{0} g_{l}, \varphi_{0} g_{l}\right) \\
& \leqq \mu+\operatorname{const}\left(l^{2}+l^{1+\frac{\beta}{2}+\varepsilon}+l^{\beta+2 s}\right) \\
& -c_{i_{0}} l^{3} \int_{\mathrm{R}_{\iota_{0}}^{3 N}} \frac{\left|g_{1}\right|^{2}}{\left|\boldsymbol{r}_{i_{0}}\right|^{\beta}} d \boldsymbol{r}_{i_{0}}+\sum_{\substack{k=1 \\
k \neq i_{0}}}^{N}\left(P_{i_{\iota}, k} \varphi_{0} g_{l}, \varphi_{0} g_{l}\right) .
\end{aligned}
$$

On the other hand, we have the following inequality

$$
P_{i_{\llcorner} k}\left(\boldsymbol{r}_{i_{s}}, \boldsymbol{r}_{k}\right) \leqq \frac{d_{i_{0} k}}{\left|\boldsymbol{r}_{i_{0}}-\boldsymbol{r}_{k}\right|^{9}}+\frac{d_{i_{k} k} R_{1}^{j^{\prime}-\beta}}{\left|\boldsymbol{r}_{i_{0}}-\boldsymbol{r}_{\kappa}\right|^{\beta^{\prime}}} .
$$

Therefore taking account of Lemma 4 , for any $\eta>0$, there exists some $l_{1}\left(1 \geqq l_{1}>0\right)$ such that

$$
\begin{aligned}
\left(P_{i_{k} k} \varphi_{0} g_{l}, \varphi_{0} g_{l}\right) \leqq \text { const } l^{\beta^{\prime}}+d_{i_{0}} l^{\beta} & \left.\int_{R_{i_{0}}^{3 v}} \frac{\left|g_{1}\right|^{2}}{\left|\boldsymbol{r}_{i_{0}}\right|^{\beta}} d r_{i_{0}}+\eta\right) \\
& \text { for any } l\left(l_{1} \geqq l>0\right) .
\end{aligned}
$$

Choosing $\eta>0$ in such a way that 


$$
\eta \sum_{\substack{k=1 \\ k \neq i_{0}}}^{N} d_{i_{0} k} \leqq \frac{1}{2}\left(c_{i_{0}}-\sum_{\substack{k=1 \\ k \neq 2_{0}}}^{N} d_{i_{0} k}\right) \int_{\mathbb{R}_{i_{0}}^{3 . V}} \frac{\left|g_{1}\right|^{2}}{\left|\boldsymbol{r}_{i_{0}}\right|^{\beta}} d \boldsymbol{r}_{i_{0}},
$$

we have

$$
\begin{aligned}
\left(H \psi_{l}, \psi_{l}\right) \leqq & +\operatorname{const}\left(l^{2}+l^{1+\frac{\beta}{2}+\varepsilon}+l^{\beta+2 \varepsilon}+l^{\beta^{\prime}}\right) \\
& -\frac{1}{2}\left(c_{i_{0}}-\sum_{\substack{k=1 \\
k \neq i_{0}}}^{N} d_{i_{0} k}\right) l^{\beta} \int_{\mathrm{R}_{i_{0}}^{3 N}} \frac{\left|g_{1}\right|^{2}}{\left|\boldsymbol{r}_{i_{0}}\right|^{\beta}} d \boldsymbol{r}_{i_{0}} .
\end{aligned}
$$

Consequently we have $\left(H \psi_{l_{2}}, \psi_{l_{2}}\right)<\mu$ for some $l_{2}\left(l_{1} \geqq l_{2}>0\right)$.

$3-r d$ step. In the case $N \geqq 2$, taking account of Lemma 2 and the 1 -st step of this proof, we have $\mu<0$.

4-th step. We shall show that there exist a countably infinite number of discrete eigenvalues. Let $N \geqq 2$. On account of the first and second steps, there exists some function $\varphi_{0}$ satisfying (4.5). We put $\psi_{l}=\varphi_{0}\left(\hat{\boldsymbol{r}}_{i_{0}}\right) g_{l}\left(\boldsymbol{r}_{i_{0}}\right)$ as before. Now we assume that there exist $s$ discrete eigenvalues of $H$. Let the discrete eigenvalues be $\left\{\lambda_{p}\right\}_{p=1, \ldots, s}$, and the eigenfunctions $\left\{u_{p}\right\}_{p=1, \ldots, s}$, where they form an orthonormal system. Putting

$$
v_{l}=\psi_{l}+\sum_{p=1}^{s} \beta_{l}^{(p)} u_{p}, \quad \text { where } \beta_{l}^{(p)}=-\left(\psi_{l}, u_{p}\right),
$$

we have

$$
\left(v_{l}, u_{p}\right)=0 \quad(p=1, \cdots, s) \text { and }\left\|v_{l}\right\|^{2}=1-\sum_{p=1}^{s}\left|\beta_{l}^{\prime p}\right|^{2} \leqq 1 .
$$

Taking account of the orthonormal relation of $\left\{u_{p}\right\}_{p=1}, s$, we also get

$$
\left(H v_{l}, v_{l}\right)=\left(H \psi_{l}, \psi_{l}\right)-\sum_{p=1}^{c} \lambda_{p}\left|\beta_{l}^{(p)}\right|^{2} .
$$

On the other hand, we have

$$
\begin{aligned}
\left|\lambda_{p}-\mu\right|\left|\beta_{l}^{(p)}\right|= & \left|\left(H u_{p}, \psi_{l}\right)-\left(u_{p}, H_{\left(i_{\nu},\right.} \psi_{l}\right)\right| \\
= & \mid\left(u_{p},\left\{\sum_{\nu=0}^{2}\left(i \frac{\partial}{\partial x_{3 i_{\nu}-\nu}}+b_{3 i_{0}-\nu}\right)^{2} \varphi_{0} g_{l}+q_{i_{0}} \varphi_{0} g_{l}\right\}\right) \\
& +\sum_{\substack{k \in 1 \\
h \neq i_{0}}}^{N}\left(u_{p}, P_{i_{\nu} k} \varphi_{0} g_{l}\right) \mid
\end{aligned}
$$


126

Jun Uchiyama

$$
\begin{aligned}
\leqq & \sum_{\nu=0}^{\prime} \mid\left(u_{p},-\varphi_{0} \frac{\partial^{2} g_{l}}{\partial x_{3 i_{0}-\nu}^{2}}\right)+\left(i \frac{\partial u_{p}}{\partial x_{3 i_{0}-\nu}}, \varphi_{0} b_{3 i_{0}-} g_{l}\right) \\
& +\left(b_{3 i_{\nu}-} u_{p}, \varphi_{0} i \frac{\partial g_{l}}{\partial x_{3 i_{\nu}-\nu}}\right)+\left(b_{3 i_{0}-\nu} u_{p}, \varphi_{0} b_{3 i_{0}-\nu} g_{l}\right) \mid \\
& +\left\|q_{i_{0}} g_{l^{\prime}}\right\|_{L^{2}\left(\mathrm{R}_{i_{0}}^{3 N}\right)}+\sum_{\substack{k=1 \\
k \neq i_{0}}}^{N}\left\|P_{i_{0} k} \varphi_{0} g_{l}\right\| \\
\leqq & \operatorname{const}\left(l^{2}+l^{1+\frac{\beta}{2}+\varepsilon}+l^{\beta+2-}+l^{\frac{\beta}{2}+\varepsilon}\right)+\left\|q_{i_{\nu}} g_{l}\right\|_{L^{2}\left(R_{i_{0}}^{3 N}\right)} \\
& +\sum_{\substack{k=1 \\
k \neq i_{0}}}^{N}\left\|P_{i_{0} k} \varphi_{0} g_{l}\right\| .
\end{aligned}
$$

However we have the following inequalities

$$
\begin{aligned}
& 0 \leqq P_{i_{0} k}\left(\boldsymbol{r}_{i_{0}}, \boldsymbol{r}_{k}\right) \\
& \begin{cases}\leqq \frac{d_{i_{0} k}}{\left|\boldsymbol{r}_{i_{0}}-\boldsymbol{r}_{k}\right|^{3}}+\frac{d_{i_{k}} R_{1}^{\beta^{\prime}-\beta} R_{2}^{r-\beta^{\prime}}}{\left|\boldsymbol{r}_{i_{0}}-\boldsymbol{r}_{k}\right|^{r}}, & \text { when } 0<\beta<\gamma<\frac{3}{2}, \\
\leqq \frac{d_{i} R_{k} R_{1}^{\beta-\beta} R_{2}^{-\beta^{\prime}}}{\left|\boldsymbol{r}_{i_{0}}-\boldsymbol{r}_{k}\right|^{r}}, & \text { when } 0<\gamma \leqq \beta<2 .\end{cases}
\end{aligned}
$$

Then on account of Lemma 3, we have

$$
\left\|P_{i_{0} k} \varphi_{0} g_{i}\right\| \leqq \operatorname{const}\left(l^{r}+l^{\circ}\right) \text {. }
$$

Therefore by means of (4.6), (4.8), (4.12), (4.13) and (4.15), we have for any $l\left(l_{1} \geqq l>0\right)$

$$
\begin{aligned}
& \left(H v_{l}, v_{l}\right) \leqq \mu+\mathrm{const}\left\{l^{2}+l^{1+\frac{\beta}{2}+\varepsilon}+l^{\beta+2}+l^{\beta^{\prime}}\right. \\
& \left.+\left(l^{2}+l^{1+\frac{\beta}{2}+\varepsilon}+l^{1+2 s}+l^{\frac{\beta}{2}+\varepsilon}+l^{*}+l^{\beta}\right)^{2}\right\} \\
& +\left(q_{i_{v}} g_{l}, g_{\imath}\right)_{\mathrm{L}^{2}\left(\mathrm{R}_{i_{0}}^{3{ }^{*}}\right)}+K\left\|q_{i_{\lrcorner}} g_{l}\right\|_{\mathrm{L}^{2}\left(\mathrm{R}_{i_{0}}^{3 \mathrm{v}}\right)}^{2} \\
& +\sum_{\substack{k=1 \\
k \neq i_{0}}}^{N} d_{i_{v} k} l^{3}\left(\int_{\mathrm{R}_{i_{0}}^{3 v}} \frac{\left|g_{j}\right|^{2}}{\left|\boldsymbol{r}_{i_{0}}\right|^{\beta}} d r_{i_{0}}+\eta\right) \text {, }
\end{aligned}
$$

where $K$ is a constant independent of $l$.

Since $q_{i_{v}}\left(\boldsymbol{r}_{i_{0}}\right)<0$ for $\left|\boldsymbol{r}_{i_{0}}\right| \geqq R_{0}$ and $g_{l}\left(\boldsymbol{r}_{i_{0}}\right) \equiv 0$ for $\left|\boldsymbol{r}_{i_{0}}\right| \leqq \frac{R_{0}}{l}$, according to (2.5) there exists for any $\eta^{\prime}>0$ some $l_{3}\left(1 \geqq l_{3}>0\right)$ such that for any $l\left(l_{3} \geqq l>0\right)$

$$
\left(q_{i_{\nu}} g_{l}, g_{l}\right)_{\mathrm{L}^{2}\left(\mathrm{R}_{i_{0}}^{3 N}\right)}+K\left\|q_{i_{\lrcorner}} g_{l}\right\|_{\mathrm{L}^{2}\left(\mathrm{R}_{i_{0}}^{3{ }^{\prime}}\right)}^{2} \leqq-c_{i_{\lrcorner}}\left(1-\eta^{\prime}\right) l^{\beta} \int_{\mathrm{R}_{i_{0}}^{3 N}} \frac{\left|g_{1}\right|^{2}}{\left|\boldsymbol{r}_{i_{0}}\right|^{\beta}} d r_{i_{0}}
$$


holds. Now let us choose, at first, $\eta^{\prime}>0$ in such a way that

$$
c_{i_{0}} \eta^{\prime} \leqq \frac{1}{2}\left(c_{i_{0}}-\sum_{\substack{k=1 \\ k \neq i_{0}}}^{N} d_{i_{0} k}\right)
$$

and in the next $\eta>0$ small enough to satisfy

$$
\eta\left(\sum_{\substack{k=1 \\ k \neq i_{0}}}^{N} d_{i_{0} k}\right) \leqq \frac{1}{4}\left(c_{i_{0}}-\sum_{\substack{k=1 \\ k \neq i_{0}}}^{N} d_{i_{0} k}\right) \int_{\mathrm{R}_{i_{0}}^{3 N}} \frac{\left|g_{1}\right|^{2}}{\left|r_{i_{0}}\right|^{\beta}} d r_{i_{0}} .
$$

Then there exists some $l_{4}\left(\min \left(l_{1}, l_{3}\right) \geqq l_{4}>0\right)$ such that

$$
\begin{aligned}
\left(H v_{l}, v_{l}\right) \leqq \mu & +\operatorname{const}\left\{l^{2}+l^{1+\frac{\beta}{2}+\varepsilon}+l^{\beta+2 \varepsilon}+l^{3^{\prime}}\right. \\
& \left.+\left(l^{2}+l^{1+\frac{\beta}{2}+\varepsilon}+l^{\beta+2 s}+l^{\frac{\beta}{2}+\varepsilon}+l^{r}+l^{5}\right)^{2}\right\} \\
& -\frac{1}{4}\left(c_{i_{0}}-\sum_{\substack{k=1 \\
k \neq i_{0}}}^{N} d_{i_{u} k}\right) l^{\beta} \int_{\mathrm{R}_{i_{0}}^{3 N}} \frac{\left|g_{1}\right|^{2}}{\left|\boldsymbol{r}_{i_{0}}\right|^{\beta}} d r_{i_{0}}
\end{aligned}
$$

holds for any $l\left(l_{4} \geqq l>0\right)$. Because of $2 \gamma>\beta$, there exists some $l_{5}\left(l_{4} \geqq l_{5}>0\right)$ such that $\left(H v_{l_{5}}, v_{l_{5}}\right)<\mu$. According to $\mu<0$ and $\left\|v_{l_{5}}\right\|_{1}^{2} \leqq 1$, we have $\left(H v_{l_{5}}, v_{l_{5}}\right)<u \leqq \mu\left\|v_{l_{5}}\right\|^{2}$. Now we put $\tilde{v}=v_{l_{0}}\left\|v_{l_{0}}\right\|^{-1}$. Then $\tilde{v}$ satisfies the following conditions

$$
\begin{aligned}
\tilde{v} \in \mathfrak{D}_{\mathrm{L}^{2}}^{2}\left(\mathrm{R}^{3 N}\right),\|\tilde{v}\|=1,\left(\tilde{v}, u_{p}\right)=0 & (p=1, \cdots, s) \\
& \text { and }(H \tilde{v}, \tilde{v})<\mu,
\end{aligned}
$$

which proves our assertion.

In the case $N=1$, we have only to make use of $g_{\imath}$ and follow the above method.

PROOF OF TheOREM 2. In the above reasoning, we have only to take as $g_{1}$ such a function that its support is contained in the cone.

Before proving Theorem 3, we shall show the following lemma.

Lemma 5. For any $\bar{\varepsilon}>0$, there exists some function $\rho(x)$ $\in \mathrm{C}_{0}^{\infty}\left(\mathrm{R}^{3}\right)(\varphi(x) \neq 0)$ such that it is identically zero in the unit ball having its center at the origin and satisfies the inequality 


$$
\int_{\mathrm{R}^{3}} \frac{|\varphi(x)|^{2}}{|r|^{2}} d x \geqq(4-\tilde{\varepsilon}) \int_{\mathrm{R} 3}|\operatorname{grad} \varphi|^{2} d x \cdot{ }^{9)}
$$

PROOF. Take a real-valued function $\rho(r)$ with the following properties,

$$
\rho(r)=\left\{\begin{array}{ll}
0, & \left(\frac{1}{2} \leqq r \leqq 1\right) \\
1, & \left(2 \leqq r \leqq \frac{5}{2}\right)
\end{array}, \quad \rho(r) \in \mathrm{C}^{\curlyvee}\left(\frac{1}{2}<r<\frac{5}{2}\right) .\right.
$$

Now we define $\zeta_{n}(r) \in \mathrm{C}_{0}^{\infty}(0 \leqq r<\infty)$ and $\varphi_{n}(x) \in \mathrm{C}_{0}^{\infty}\left(\mathrm{R}^{3}\right)$ as follows,

$$
\begin{aligned}
& \zeta_{n}(r)= \begin{cases}0, & (0 \leqq r \leqq 1) \\
\rho(r), & (1 \leqq r \leqq 2) \\
1, & (2 \leqq r \leqq 2 n), \\
\rho\left(4-\frac{r}{n}\right), & (2 n \leqq r \leqq 3 n) \\
0, & (r \geqq 3 n)\end{cases} \\
& \varphi_{n}(x)=\frac{\zeta_{n}(r)}{\sqrt{ } \frac{r}{r},} \text { where } r=|r|=\sqrt{{x_{1}{ }^{2}+x_{2}{ }^{2}+x_{3}{ }^{2}}^{2}}
\end{aligned}
$$

Then we have

$$
\left|\operatorname{grad} \varphi_{n}\right|^{2}=\left|\frac{d \varphi_{n}}{d r}\right|^{2}=\frac{1}{4} \cdot \frac{\zeta_{n}(r)^{2}}{r^{3}}-\frac{\zeta_{n}(r) \cdot \zeta_{n}^{\prime}(r)}{r^{2}}+\frac{\zeta_{n}^{\prime}(r)^{2}}{r} .
$$

Thus

$$
\int_{\mathrm{R}^{3}} \frac{\left|\varphi_{n}(x)\right|^{2}}{r^{2}} d x=\int_{\mathrm{R}^{3}} \frac{\zeta_{n}(r)^{2}}{r^{3}} d x=4 \int_{\mathrm{R}^{3}}\left|\operatorname{grad} \varphi_{n}\right|^{2} d x-16 \pi \int_{1}^{3 n} \zeta_{n}^{\prime}(r)^{2} \cdot r d r .
$$

On the other hand, we have

$$
\begin{aligned}
\int_{1}^{3 n} \zeta_{n}^{\prime}(r)^{2} r d r & =\int_{1}^{2} \rho^{\prime}(r)^{2} r d r+\int_{2 n}^{3 n} \rho^{\prime}\left(4-\frac{r}{n}\right)^{2} \cdot \frac{r}{n^{2}} d r \\
& =\int_{1}^{2} \rho^{\cdot}(r)^{2} r d r+\int_{2}^{3} \rho^{\prime}(4-r)^{2} r d r,
\end{aligned}
$$

and

9) Taking account of the well-known inequality

$$
\int_{\mathrm{R}^{3}} \frac{|\varphi(x)|^{2}}{|\boldsymbol{r}|^{2}} d x \leqq 4 \int_{\mathrm{R}^{3}}|\operatorname{grad} \varphi|^{2} d x \quad\left(\varphi \in \mathrm{C}_{0}^{\infty}\left(\mathrm{R}^{3}\right)\right),
$$

Lemma 4 shows that in the above estimate the constant 4 is best. 


$$
\int_{\mathrm{R}^{3}} \frac{\left|\varphi_{n}(x)\right|^{2}}{r^{2}} d x=4 \pi \int_{0}^{\infty} \frac{\zeta_{n}(r)^{2}}{r} d r \geqq 4 \pi \int_{2}^{2 n} \frac{d r}{r}=4 \pi \log n
$$

Then choosing $n_{0}$ sufficiently large, we have

$$
\begin{aligned}
& \int_{\mathrm{R}^{3}} \frac{\left|\varphi_{n_{0}}(x)\right|^{2}}{r^{2}} d x \\
& \quad=4\left\{1+16 \pi \int_{1}^{3 n_{0}} \zeta_{n_{0}}^{\prime}(r)^{2} r d r\left(\int_{\mathrm{R}^{3}} \frac{\left|\varphi_{n_{0}}(x)\right|^{2}}{r^{2}} d x\right)^{-1}\right\}^{-1} \int_{\mathrm{R}^{3}}\left|\operatorname{grad} \varphi_{n_{0}}(x)\right|^{2} d x \\
& \quad \geqq(4-\tilde{\varepsilon}) \int_{\mathrm{R}^{3}}\left|\operatorname{grad} \varphi_{n_{0}}\right|^{2} d x,
\end{aligned}
$$

which shows that $\varphi_{n_{0}}(x) \in \mathrm{C}_{0}^{\infty}\left(\mathrm{R}^{3}\right)$ satisfies the property required.

Now we shall prove Theorem 3 .

PROOF. We can choose $4>\tilde{\varepsilon}>0$ small enough to satisfy

$$
c_{i_{0}}-\sum_{\substack{k=1 \\ k \neq i_{0}}}^{N} d_{i_{0} k}-\frac{1}{4-\tilde{\varepsilon}}>0 .
$$

Then we take $\varphi(x) \in \mathrm{C}_{0}^{\infty}\left(\mathrm{R}^{3}\right)$ satisfying Lemma 5 for $\tilde{\varepsilon}>0$ and put

$$
g_{1}\left(x_{1}, x_{2}, x_{3}\right)=R_{0}^{-\frac{3}{2}} \cdot\|\varphi\|_{\mathrm{L}^{2}\left(\mathrm{R}^{3}\right)}^{-1} \cdot \varphi\left(\frac{x_{1}}{R_{0}}, \frac{x_{2}}{R_{0}}, \frac{x_{3}}{R_{0}}\right)
$$

which satisfies (4.1) and (4.2). Let $g_{l}(x)=l^{\frac{3}{2}} g_{1}\left(l x_{1}, l x_{2}, l x_{3}\right)$ as before. Now we shall follow the proof of Theorem 1. By means of Lemma 5, we have the following inequality in place of (4.3)

$$
\begin{aligned}
\left(4.3^{\prime}\right)\left(H g_{l}, g_{l}\right) \leqq & \operatorname{const}\left(l^{2+\varepsilon}+l^{2+2 \varepsilon}\right)-c_{i_{0}} l^{2} \int_{\mathrm{R}^{3}} \frac{\left|g_{1}(x)\right|^{2}}{|\boldsymbol{r}|^{2}} d x \\
& +l^{2} \int_{\mathrm{R}^{3}}\left|\operatorname{grad} g_{1}\right|^{2} d x \\
\leqq & \operatorname{const}\left(l^{2+3}+l^{2+2-}\right)-\left(c_{i_{0}}-\frac{1}{4-\tilde{\varepsilon}}\right) l^{2} \int_{\mathrm{R}^{3}} \frac{\left|g_{1}(x)\right|^{2}}{|\boldsymbol{r}|^{2}} d x,
\end{aligned}
$$

which shows that the assertion of the 1-st step in the proof of Theorem 1 is also true in this case.

Secondly by virtue of Lemma 5 and (4.8), we have the following estimate in place of (4.6). 
(4. $\left.6^{\prime}\right) \quad\left(H \psi_{l}, \psi_{l}\right) \leqq \mu+\operatorname{const}\left(l^{2+\varepsilon}+l^{2+2 \varepsilon}+l^{\beta^{\prime}}\right)+\left\{\int_{\mathrm{R}_{i_{0}}^{3 N}}\left|\operatorname{grad} g_{1}\right|^{2} d r_{i_{0}}\right.$

$$
\begin{aligned}
& \left.\quad-c_{i_{0}} \int_{\mathrm{R}_{i_{0}}^{3 N}}-\frac{\left|g_{1}\right|^{2}}{\left|r_{i_{0}}\right|^{2}} d r_{i_{0}}+\sum_{\substack{k=1 \\
k \neq i_{0}}}^{N} d_{i_{\diamond} k}\left(\int_{\mathrm{R}_{i_{0}}^{3 N}} \frac{\left|g_{1}\right|^{2}}{\left|r_{i_{0}}\right|^{2}} d r_{i_{0}}+\eta\right)\right\} l^{2} \\
& \leqq \\
&
\end{aligned}
$$

Then choosing $\eta>0$ in such a way that

$$
\eta \sum_{\substack{k=1 \\ k \neq i_{0}}}^{N} d_{\left.i_{\lrcorner}\right\rfloor} \leqq \frac{1}{2}\left(c_{i_{\lrcorner}}-\sum_{\substack{k=1 \\ k \neq i_{0}}}^{N} d_{i_{0} k}-\frac{1}{4-\tilde{\varepsilon}}\right) \int_{\mathrm{R}_{i_{0}}^{3 N}} \frac{\left|g_{1}\right|^{2}}{\left|r_{i_{0}}\right|^{2}} d r_{i_{0}},
$$

we have

$$
\begin{aligned}
\left(H \psi_{l}, \psi_{l}\right) \leqq & \mu+\operatorname{const}\left(l^{2+s}+l^{2+2}+l^{\beta^{\prime}}\right) \\
& -\frac{1}{2}\left(c_{i_{0}}-\sum_{\substack{h=1 \\
k \neq i_{0}}}^{N} d_{i_{\mathrm{v}} \varepsilon}-\frac{1}{4-\tilde{\varepsilon}}\right) \int_{\mathrm{R}_{i_{0}}^{3 N}} \frac{\left|g_{1}\right|^{2}}{\left|r_{i_{0}}\right|^{2}} d r_{i_{0}},
\end{aligned}
$$

which shows the assertion of the second step in the proof of Theorem 1 is also true in this case.

Last of all, taking account of Lemma 5, (4.8) and (4.17), we have the following inequality in place of (4.16).

$$
\begin{aligned}
& \left(H v_{l}, v_{l}\right) \leqq \mu+\mathrm{const}\left\{l^{2+\varepsilon}+l^{2+2 \varepsilon}+l^{\beta^{\prime}}+\left(l^{2}+l^{2+\varepsilon}+l^{2+2 \varepsilon}+l^{1+\varepsilon}+l^{r}\right)^{2}\right\} \\
& +\left\{\int_{\mathrm{R}_{i_{0}}^{3 . V}}\left|\operatorname{grad} g_{1}\right|^{2} d r_{i_{j}}-c_{i_{0}}\left(1-\eta^{\prime}\right) \int_{\mathrm{R}_{i_{0}}^{3 N}} \frac{\left|g_{1}\right|^{2}}{\left|r_{i_{0}}\right|^{2}} d r_{i_{0}}\right. \\
& \left.+\sum_{\substack{k=1 \\
k \neq i_{0}}}^{N} d_{i_{0} k}\left(\int_{\mathrm{R}_{i_{0}}^{3 N}} \frac{\left|g_{1}\right|^{2}}{\left|r_{i_{0}}\right|^{2}} d r_{i_{0}}+\eta\right)\right\} l^{2} \\
& \leqq \mu+\operatorname{const}\left\{l^{2+\varepsilon}+l^{2+2 \varepsilon}+l^{\beta^{\prime}}+\left(l^{2}+l^{2+\varepsilon}+l^{2+2 \varepsilon}+l^{1+\varepsilon}+l^{r}\right)^{2}\right\} \\
& -\left(c_{i_{0}}-\sum_{\substack{k=1 \\
k \neq \imath_{0}}}^{N} d_{i_{\iota} k}-\frac{1}{4-\tilde{\varepsilon}}\right) l^{2} \int_{\mathrm{R}_{i_{0}}^{3 N}} \frac{\left|g_{1}\right|^{2}}{\left|r_{i_{0}}\right|^{2}} d r_{i_{J}} \\
& +c_{i_{0}} \eta^{\prime} l^{2} \int_{\mathrm{R}_{i_{0}}^{3 N}} \frac{\left|g_{1}\right|^{2}}{\left|r_{i_{0}}\right|^{2}} d r_{i_{0}}+\left(\sum_{\substack{k=1 \\
h \neq i_{0}}}^{N} d_{i_{0} k}\right) \eta l^{2} .
\end{aligned}
$$

Then choosing $\eta^{\prime}>0$ and $\eta>0$ to satisfy 


$$
\begin{aligned}
& c_{i_{0}} \eta^{\prime} \leqq \frac{1}{2}\left(c_{i_{0}}-\sum_{\substack{k=1 \\
k=i_{0}}}^{N} d_{i_{v} k}-\frac{1}{4-\tilde{\varepsilon}}\right) \text { and } \\
& \eta \sum_{\substack{k=1 \\
k \neq i_{0}}}^{N} d_{i_{v} k} \leqq \frac{1}{4}\left(c_{i_{0}}-\sum_{\substack{k=1 \\
k \neq i_{0}}}^{N} d_{i_{0} k}-\frac{1}{4-\tilde{\varepsilon}}\right) \int_{\mathrm{R}_{02}^{3 N}}-\mid \frac{\left.g_{1}\right|^{2}}{\left|r_{i_{0}}\right|^{2}} d r_{i_{0}},
\end{aligned}
$$

we have

$$
\begin{aligned}
\left(H v_{l}, v_{l}\right) \leqq & \mu+\operatorname{const}\left\{l^{2+\varepsilon}+l^{2+2 \varepsilon}+l^{\beta^{\prime}}+\left(l^{2}+l^{2+\varepsilon}+l^{2+2 \varepsilon}+l^{1+\varepsilon}+l^{r}\right)^{2}\right\} \\
& -\frac{1}{4}\left(c_{i_{0}}-\sum_{\substack{k=1 \\
k \neq 2_{0}}}^{N} d_{i_{0} k}-\frac{1}{4-\tilde{\varepsilon}}\right) l^{2} \int_{\mathrm{R}_{c 0}^{3 N}} \frac{\left|g_{1}\right|^{2}}{\left|r_{i_{0}}\right|^{2}} d r_{i_{0}},
\end{aligned}
$$

which shows that the same assertion as that of the 4-th step in the proof of Theorem 1 is true in our case.

Remark 2. In the case when the masses of the particles of the system are not all the same, applying a suitable linear transformation such that $\boldsymbol{r}_{k}^{\prime}=\frac{1}{\omega_{k}} \boldsymbol{r}_{k}\left(\omega_{k}>0\right)$, we see easily that we have only to consider the system under the following assumption. Namely, we replace the conditions (2.2) and (2.4) by the conditions

$\left(2.4^{\prime}\right) \quad 0 \leqq P_{k h}\left(\boldsymbol{r}_{k}, \boldsymbol{r}_{h}\right) \begin{cases}\leqq \frac{d_{k h} R_{1}^{\beta^{\prime}-\beta} R_{2}^{r-\beta^{\prime}}}{\left|\omega_{k} \boldsymbol{r}_{k}-\omega_{h} \boldsymbol{r}_{h}\right|^{r}} & \text { for }\left|\omega_{k} r_{k}-\omega_{h} r_{h}\right| \leqq R_{2}, \\ \leqq \frac{d_{k h} R_{1}^{\beta^{\prime}-\beta}}{\left|\omega_{k} r_{k}-\omega_{h} r_{h}\right|^{\beta^{\prime}}} & \text { for } \quad R_{2} \leqq\left|\omega_{k} r_{k}-\omega_{h} r_{h}\right| \leqq R_{1}, \\ \leqq \frac{d_{k h}}{\left|\omega_{k} \boldsymbol{r}_{k}-\omega_{h} \boldsymbol{r}_{h}\right|^{\beta}} & \text { for }\left|\omega_{k} r_{k}-\omega_{h} r_{h}\right| \geqq R_{1} .\end{cases}$

Then we can get the same results as that of theorem 1 . And in this case replacing $\left(\mathrm{C}-4^{\prime}\right)$ by the condition

$$
c_{k}-\sum_{\substack{h=1 \\ h \neq k}}^{N} d_{k h}>\frac{1}{4} \cdot \omega_{k}^{2},
$$

we can easily obtain the same result as that of Theorem 3 .

In conclusion, the writer wishes to express his sincere 
gratitude to Professors S. Mizohata, T. Ikebe and K. Mochizuki for their enduring encouragements and kind instructions.

\section{REFERENCES}

[1] Jörgens, K. : Über das wesentliche Spectrum elliptischer Differential-operatoren vom Schrödinger-Typ. Institut für Angewandte Mathematik, Universität Heidelberg, 1965.

[2] Žislin, G. M. : Discussion of the spectrum of the Schrödinger operator for manyparticle system. Trudy Mosk. Mat. Obšč.9, 82-120, (1960) (in Russian). 